\title{
Efficient Computation of PDF-Based Characteristics from Diffusion MR Signal
}

\author{
Haz-Edine Assemlal, David Tschumperlé, and Luc Brun \\ GREYC (CNRS UMR 6072), 6 Bd Maréchal Juin, 14050 Caen Cedex, France*
}

\begin{abstract}
We present a general method for the computation of PDFbased characteristics of the tissue micro-architecture in MR imaging. The approach relies on the approximation of the MR signal by a series expansion based on Spherical Harmonics and Laguerre-Gaussian functions, followed by a simple projection step that is efficiently done in a finite dimensional space. The resulting algorithm is generic, flexible and is able to compute a large set of useful characteristics of the local tissues structure. We illustrate the effectiveness of this approach by showing results on synthetic and real MR datasets acquired in a clinical time-frame.
\end{abstract}

\section{Introduction}

Diffusion-weighted imaging (DWI) uses a pulse gradient spin echo sequence [1] in order to provide in-vivo images of the water molecule diffusion. The acquired apparent diffusion coefficient images (ADC) give a pointwise measure of the magnetic resonance (MR) diffusion signal attenuation in the tissue microstructure. These images provide thus valuable information to diagnose early stages of stroke, brain diseases or neurological disorders [2]. Assuming that the water displacement probability is constrained by the nerve fiber structures, it is possible to characterize the local structures of fibers in each acquired image voxel. Indeed, when the diffusion gradient pulse duration $\delta$ is negligible compared to diffusion time $\Delta$, the MR signal attenuation $E$ defined in Q-Space is related to the average displacement probability $P$ by the Fourier transform [3]

$$
P(\mathbf{p})=\int_{\mathbf{q} \in \mathbb{R}^{3}} E(\mathbf{q}) \exp (-2 \pi i \mathbf{q p}) d \mathbf{q}, \quad \text { with } E(\mathbf{q})=\frac{S(\mathbf{q})}{S_{0}},
$$

where $\mathbf{p}$ is the displacement vector and $\mathbf{q}$ stands for the diffusion wave-vector of the Q-Space. The symbols $S(\mathbf{q})$ and $S_{0}$ respectively denote the diffusion signal at $\mathbf{q}$ and the baseline image at $\mathbf{q}=0$. Diffusion Tensor Imaging (DTI) 4 models the MR signal attenuation $E$ with a Gaussian function $E(\mathbf{q})=\exp \left(-4 \pi^{2} \Delta \mathbf{q}^{T} D \mathbf{q}\right)$ where the symbol $D$ denotes the ADC and is modeled as a second-order tensor. Consequently, DTI can only map a single fiber orientation inside a voxel and fails in voxels with orientational heterogeneity [5].

\footnotetext{
^ Many thanks to the laboratory GIN Cyceron, France for providing in-vivo data.
} 
Eq.(11) naturally suggests to use the Fourier transform to numerically estimate the PDF (Probability Density Function). This technique known as diffusion spectrum imaging (DSI) [6] is not clinically feasible due to the huge acquisition time required to retrieve the whole Q-Space coefficients. As a result of DSI constraints, High Angular Resolution Diffusion Imaging (HARDI) [7] comes as an interesting alternative and suggests to sample the signal on a single sphere of the Q-Space. Among these studies, Q-Ball imaging (QBI) 899 focuses on angular information of the PDF and so approximates the radial integral of the PDF, known as the orientation density function (ODF) using the Funk-Radon Transform (FRT). The fiber orientation distribution (FOD) method computes the whole PDF volume by introducing a prior on either angular or radial MR signal or both 5[1011. Some approaches assumes a mono-exponential radial MR decay and propose analytical solutions to the Fourier transform, as in the generalized DTI method based on the Fick's diffusion law [12], and in the diffusion orientation transform (DOT) using the Hankel transform [13].

Previously mentioned methods based on HARD images use a single radial acquisition and have thus to assume strong priors on the radial behavior of the signal. Nonetheless, sampling schemes on several spheres in the Q-Space have been proposed recently $13|14| 15$. Since the number of samples remains still too low to allow a precise Fourier transform, other methods rather consider an approximation of the MR signal radial attenuation by a multi-exponential function 1314. Note that these methods use a larger set of data but are still based on a priori models of the radial behavior of the input signal. In section 2 , we present a new and flexible method to compute any characteristic on the PDF, based on a "model-free" estimation of the signal. Then, we illustrate in section 3 validation results on both numerical and real human data-sets. Finally, we draw conclusions of the proposed approach in section 4.

\section{Estimation of PDF-Based Characteristics}

The estimation of the PDF from Eq.(11) is a trade-off between the number of samples and the prior on the MR signal, so we introduce an orthonormal basis based on spherical harmonics and Gaussian-Laguerre polynomials in which we expect the signal to be sparse. As a consequence low-order truncation assumes radial Gaussian behavior as in 13/14 and high order truncation order provide a model-free estimation. Note that the truncation order is restricted by the number of data samples in the acquisition. For convenience, a list of common notations used in this paper is reminded in Table 1

Spherical Polar Fourier Expansion: Since the samples are generally acquired on one or several spheres of the Q-Space, our orthonormal basis is defined as a combination of angular and radial elementary functions expressed in spherical coordinates. Here, we expand the MR signal attenuation $E$ as a series in a spherical orthonormal basis named Spherical Polar Fourier (SPF) [16]

$$
E(\mathbf{q})=\frac{S(\mathbf{q})}{S(0)}=\sum_{n=0}^{\infty} \sum_{l=0}^{\infty} \sum_{m=-l}^{l} a_{n l m} R_{n}(|\mathbf{q}|) y_{l}^{m}\left(\frac{\mathbf{q}}{|\mathbf{q}|}\right),
$$


Table 1. A list of major notations used in this paper

\begin{tabular}{ll|ll} 
Symbol & Description & Symbol & Description \\
\hline PDF & Probability Density Function & $\mathbf{p}, \mathbf{k}$ & Displacement vectors in $\mathbb{R}^{3}$ \\
ODF & Orientation Density Function & $P(\mathbf{p})$ & Average displacement probabilities \\
FRT & Funk-Radon Transform & $\mathbf{q}$ & Diffusion space vector in $\mathbb{R}^{3}$ \\
SH & Spherical Harmonics & $S(\mathbf{q})$ & MR signal at diffusion gradient $\mathbf{q}$ \\
SPF & Spherical Polar Fourier & $E(\mathbf{q})$ & MR signal attenuation $S(\mathbf{q}) / S(0)$ \\
$a_{n l m}$ & SPF expansion coefficient & $\mathcal{G}(\mathbf{k})$ & PDF characteristic at point $\mathbf{k}$ \\
& at order $n, l$ and $m$ & $h_{\mathbf{k}}(\mathbf{p})$ & projection function of $\mathcal{G}(\mathbf{k})$
\end{tabular}

where $a_{n l m}$ are the expansion coefficients, $y_{l}^{m}$ are real spherical harmonics (SH), and $R_{n}$ is an orthonormal radial basis function.

The angular part of the signal is reconstructed by elementary angular functions based on the Spherical Harmonics basis $Y_{l}^{m}$. The complex SH $Y_{l}^{m}$ form an orthonormal basis for functions defined on the single sphere and are related to the solution of the Laplace's equation in spherical coordinates. For this reason, they have been widely used in dMRI [17. Indeed, as the diffusion signal exhibits real and symmetry properties, the use of a subset of the complex basis made of real and symmetric SH $y_{l}^{m}$ strengthen the robustness of the reconstruction to signal noise and reduces the number of required coefficients [17 : an expansion to order $n<=N$ and $l<=L$ only involves $(N+1)(L+1)(L+2) / 2$ coefficients.

The radial part of the signal is reconstructed by the elementary radial functions $R_{n}$. A sparse representation of the radial signal should approximate it in a few radial order $N$. Several studies [18|19] has shown that the signal decay $E$ appeared to be a composition of Gaussian functions, the number of functions being determined by the wave-vector norm $|\mathbf{q}|$. In practice, several authors 20|21. reported that a bi-exponential function is sufficient to fit $E$, suggesting a model with a slow and a fast free diffusion in correspondence to the intra and extra cellular compartments. However the spatial resolution of voxels is such that it forms a complex physical system which contains numerous cells with various characteristics. Therefore a direct relationship between the bi-exponential attenuation and the intra-extra cellular diffusion is not obvious and has yet to be investigated [20]. As a result, the origin of the signal attenuation is still unclear and the bi-exponential model does not necessarily describe the diffusion system accurately [19. Based on these observations, we propose a general estimation of the radial part of the signal attenuation $E$ using the normalized generalized Gaussian-Laguerre polynomials basis $R_{n}$ (also referred as Gaussian-Type Orbitals (GTO) in the molecular crystallography community [16/22]):

$$
R_{n}(|\mathbf{q}|)=\left[\frac{2}{\gamma^{3 / 2}} \frac{n !}{\Gamma(n+3 / 2)}\right]^{1 / 2} \exp \left(-\frac{|\mathbf{q}|^{2}}{2 \gamma}\right) L_{n}^{1 / 2}\left(\frac{|\mathbf{q}|^{2}}{\gamma}\right),
$$

where $\gamma$ denotes the scale factor and $L_{n}^{(\alpha)}$ are the generalized Laguerre polynomials. The Gaussian decay arises from the normalization of the Laguerre polynomials in spherical coordinates. Note that QBI 8 19] and DOT [13] methods can be expressed in this approach with respectively $R_{n}(|\mathbf{q}|)=\delta\left(\left|\mathbf{q}^{\prime}\right|-|\mathbf{q}|\right)$ and 
Table 2. A non-exhaustive list of some PDF characteristics $\mathcal{G}$ and their projection function $h_{\mathbf{k}}$ at point $\mathbf{k}$. FRT stands for the Funk-Radon Transform used in QBI, where $J_{0}$ is the Bessel function of the first kind and $\left|\mathbf{q}^{\prime}\right|$ is the radius of the q-ball shell. ISO stands for isoprobability profiles. SD and FD respectively stands for slow and fast diffusion, where $\left|\mathbf{p}^{\prime}\right|$ is the radius limit between intra and extra cellular diffusion.

\begin{tabular}{|l||c|c|c|c|c|}
\hline $\mathcal{G}$ & ODF & FRT & ISO & SD & FD \\
\hline$h_{\mathbf{k}}(\mathbf{p})$ & $\delta\left(1-\frac{|\mathbf{p} \cdot \mathbf{k}|}{|\mathbf{p}||\mathbf{k}|}\right)$ & $J_{0}\left(2 \pi\left|\mathbf{q}^{\prime}\right||\mathbf{p}|\right)$ & $\delta(\mathbf{k})$ & ODF if $|\mathbf{p}|<\left|\mathbf{p}^{\prime}\right|$ & $0 \quad$ if $|\mathbf{p}|<\left|\mathbf{p}^{\prime}\right|$ \\
0 & if $|\mathbf{p}|>\left|\mathbf{p}^{\prime}\right|$ & ODF if $|\mathbf{p}|>\left|\mathbf{p}^{\prime}\right|$ \\
\hline
\end{tabular}

$R_{n}(|\mathbf{q}|)=j_{n}\left(2 \pi|\mathbf{q}|\left|\mathbf{p}^{\prime}\right|\right) \delta_{n l}$, where the symbols $\left|\mathbf{q}^{\prime}\right|$ and $\left|\mathbf{p}^{\prime}\right|$ are constant values defined in Table 2 .

Spherical Polar Fourier (SPF) is a set of functions which forms a complete, orthogonal basis; consequently the square error between a function and its expansion converges to zero as $N$ and $L$ become infinite. We fit the signal to the SPF by a damped least square minimization and best fitting coefficients $a_{n l m}$ are given by a regularized Moore-Penrose pseudo-inverse scheme:

$$
\tilde{A}=\underset{\tilde{A}}{\arg \min }|\tilde{E}-\tilde{M} \tilde{A}|^{2}=\left(\tilde{M}^{T} \tilde{M}+\lambda_{l} \tilde{L}+\lambda_{n} \tilde{N}\right)^{-1} \tilde{M}^{T} \tilde{E}
$$

where $\tilde{M}=\left(R_{n}\left(\left|\mathbf{q}_{j}\right|\right) y_{l}^{m}\left(\frac{\mathbf{q}_{j}}{\left|\mathbf{q}_{j}\right|}\right)\right)_{n l m \times j \in \mathbb{N}^{3} \times \mathbb{N}}$ denotes the SPF basis matrix and $\tilde{E}, \tilde{A}$ respectively denote the vectors $\left(E\left(\mathbf{q}_{1}\right), \ldots, E\left(\mathbf{q}_{n s}\right)\right)^{T}$ and $\left(a_{000}, \ldots, a_{N L L}\right)^{T}$. The matrix $\tilde{L}$ and $\tilde{N}$ are the regularization matrix with entries $l^{2}(l+1)^{2}$ and $n^{2}(n+1)$ along their diagonal, which respectively penalizes high degrees of the angular part and radial part of SPF in the estimation under the assumption that they are likely to capture noise. The symbols $\lambda_{l}$ and $\lambda_{n}$ respectively denote angular and radial weight regularization term.

Projection: As we have a continuous representation of $E$ from the SPF coefficients, let $\mathcal{G}(\mathbf{k})=\int P(\mathbf{p}) h_{\mathbf{k}}(\mathbf{p}) d \mathbf{p}$ be a characteristic $\mathcal{G}$ of the PDF at point $\mathbf{k}$, where $h_{\mathbf{k}}$ denotes a projection function at point $\mathbf{k}$. Table 2 represents several popular characteristics $\mathcal{G}$ which can be evaluated using this computational scheme. A natural way to retrieve $\mathcal{G}$ would be to reconstruct $E$ from the SPF coefficients, compute an Fast Fourier Transform (FFT) and finally calculate $\mathcal{G}$ on the PDF volume; however such a scheme would induce cumbersome computations and raise numerical accuracy issues. So, any characteristic $\mathcal{G}$ defined from Eq.(5) can alternatively be computed directly from the SPF coefficients. Indeed, since the SPF are an orthonormal basis the following relation holds:

$$
\mathcal{G}(\mathbf{k})=\int_{\mathbf{p} \in \mathbb{R}^{3}} P(\mathbf{p}) h_{\mathbf{k}}(\mathbf{p}) d \mathbf{p}=\int_{\mathbf{q} \in \mathbb{R}^{3}} E(\mathbf{q}) H_{\mathbf{k}}(\mathbf{q}) d \mathbf{q}=\sum_{n l m}^{\infty} a_{n l m} b_{n l m}^{\mathbf{k}}
$$

where $H_{\mathbf{k}}$ is the inverse Fourier transform of $h_{\mathbf{k}}$ and $a_{n l m}, b_{n l m}^{\mathbf{k}}$ respectively denote the SPF expansion of $E$ and $H_{\mathbf{k}}$. Therefore, the computation of $\mathcal{G}(\mathbf{k})$ which is an integration over an entire volume simply turns into a very fast dot product between two vectors of SPF coefficients. 


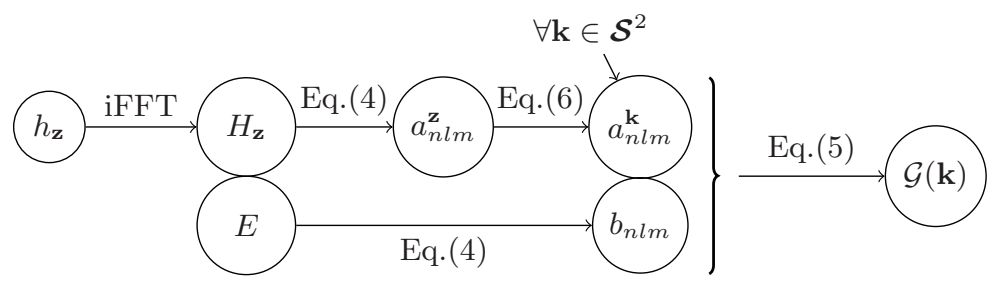

Fig. 1. Algorithm overview for the computation of PDF characteristics $\mathcal{G}$ at point $\mathbf{k}$, defined on the single sphere $\mathcal{S}^{2}$

Characteristics Defined on the Sphere: At this point, we have to compute the inverse FFT of $h_{\mathbf{k}}$ and its SPF expansion for each probability vector $\mathbf{k}$. All these computations are required only once and can be stored in computer memory for later use. An alternative faster scheme arises for characteristics which are angular-dependent only; i.e. $\mathbf{k} \in \mathcal{S}^{2}$ where $\mathcal{S}^{2}$ is the sphere domain. Note that all characteristics $\mathcal{G}$ defined in table 2 satisfy this constraint. In this case, we can use the rotation property of $\mathrm{SH}$ which states that a rotation of a $\mathrm{SH}$ can be expressed as a linear combination of $\mathrm{SH}$ of the same degree $l$. As a consequence, a rotation of any linear combination of SPF can be expressed as:

$$
a_{n l m}^{\prime}=\sum_{m^{\prime}=-l}^{l} a_{n l m^{\prime}} R_{m m^{\prime}}^{(l)}(\alpha, \beta, \gamma)
$$

where $a_{n l m}$ and $a_{n l m}^{\prime}$ are respectively a linear combination of SPF and its rotation. $R_{m m^{\prime}}^{(l)}$ stands for the real Wigner rotation matrix expressed in terms of Euler angles $(\alpha, \beta, \gamma)$ in $z y z$ convention (see [23] for more technical details). As we want to compute the projection on a single sphere, we set $(\alpha, \beta, \gamma)=(\phi, \theta, 0)$, where $\phi \in[0,2 \pi)$ and $\theta \in[0, \pi]$ follow spherical coordinates from physics convention. In this case, $h_{\mathbf{k}}$ has to be constructed only once with $\theta=\phi=0$, i.e. $\mathbf{k}$ initially taken as the z-axis unitary vector $\mathbf{z}$. Schematic description of the algorithm for characteristics defined on the single sphere is given at Fig 1 .

\section{$3 \quad$ Experiments}

In this section, we present results of our method on both synthetic simulations and real human brain data-sets. We focus on a comparison of ODF computed with QBI method as proposed in [9] and with our method using either $\mathcal{G}=\mathrm{FRT}$ and $\mathcal{G}=\mathrm{ODF}$ characteristics. Additionally, we show the estimation improvements along with the number of diffusion MR signal samples.

Numerical Simulations: We have applied the above scheme to the simulations of a single fiber and crossing fiber configurations. The following synthetic multiexponential model was used to generate data, $E(\mathbf{q})=\sum_{k=1}^{N_{b}} f_{k} \exp \left(-\mathbf{q}^{T} D_{k} \mathbf{q}\right)$ where $\sum_{k=1}^{N_{b}} f_{k}=1$. The symbol $N_{b}$ stands for the number of fibers and $D_{k}$ 


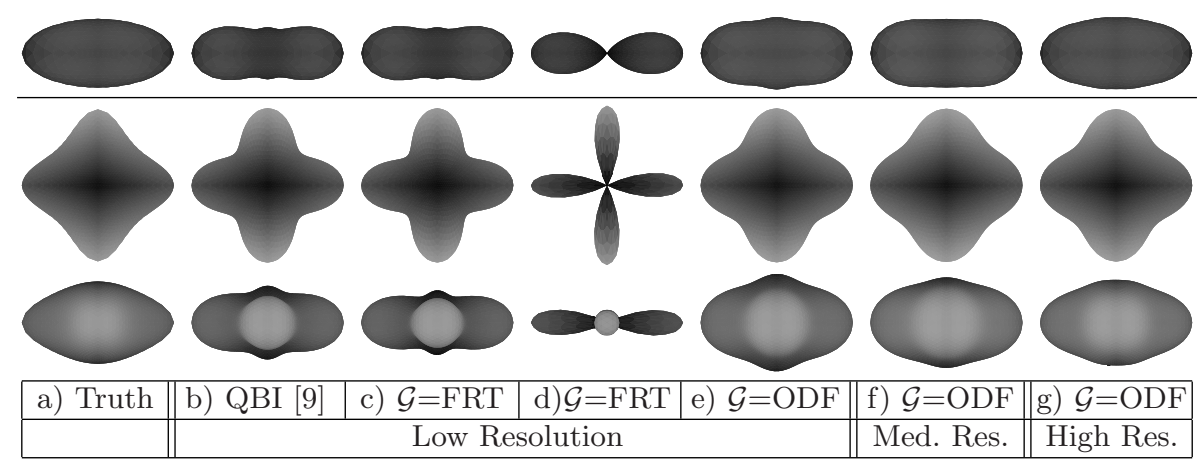

Fig. 2. ODF comparisons: ODF versus $\mathrm{QBI}$ and $\mathrm{ODF}$ at low resolution versus ODF at high resolution. The first line corresponds to a single fiber direction $N_{b}=1$. The second and third lines correspond to crossing fibers $N_{b}=2$ respectively in face and profile view. From the left to the right: (a) True ODF computed analytically, (b) ODF given by QBI [9], (c) simulation of QBI with our method, (d) FRT with higher $q^{\prime}$, (e-g) ODF estimations given by our method, with increasing number of samples.

is a $3 \times 3$ symmetric definite positive matrix defining the diffusion anisotropy. Diffusion images were synthesized following 3 sampling protocols: low resolution ( 1 shell $\left.b=3000 \mathrm{~s} / \mathrm{mm}^{2}\right)$, medium resolution ( 2 shells $b=\{1000,3000\} \mathrm{s} / \mathrm{mm}^{2}$ ) and high resolution (5 shells $b=\{500,1000,1700,2400,3000\} \mathrm{s} / \mathrm{mm}^{2}$ ) along with a single baseline image acquired at $b=0 \mathrm{~s} / \mathrm{mm}^{2}$. Each shells is composed of 42 directions along the edges of a subdivided icosahedron. Estimation parameters were chosen empirically for each sampling protocol: low resolution $\{N=0$, $\left.L=4, \gamma=100, \lambda_{N}=0, \lambda_{L}=10^{-6}\right\}$, medium resolution $\{N=1, L=4, \gamma=70$, $\left.\lambda_{N}=\lambda_{L}=0\right\}$, high resolution $\left\{N=4, L=6, \gamma=50, \lambda_{N}=\lambda_{L}=10^{-9}\right\}$. The scale factor $\gamma$ was set empirically so that the decay of the basis atoms have the same scale as the sampled data. Let $x=R_{n}\left(\left|\mathbf{q}^{\prime}\right|\right) / R_{n}(0)$ where $x \in[0,1]$. After some algebra, this leads to $\gamma \approx\left(\left|\mathbf{q}^{\prime}\right|^{2} \sqrt{\pi} n !\right) /\left(4 \Gamma\left(n+\frac{3}{2}\right) \log \left(\frac{1}{x L_{n}^{1 / 2}(0)}\right)\right)$. In this work, $\left|\mathbf{q}^{\prime}\right|=30 \mathrm{~mm}^{-1}, x=E\left(\left|\mathbf{q}^{\prime}\right|\right) / E(0)=0.01$ and $n=N$.

Results of Fig 2 (b-c) validate that our method can successfully reproduce the ODF obtained with the QBI approach, with a standard HARD acquisition. Note that the resulting ODF is a sharp approximation of the true ODF. Besides, an extrapolation of QBI with a higher $\left|\mathbf{q}^{\prime}\right|$ value logically gives even sharper ODF as illustrated in Fig 2(d). With the same data-set, the ODF of our method Fig 2(e) gives a more precise representation of the true ODF. This is due to the Gaussian assumption of our model at low radial $N$ order which enables to better fit the synthetic data. We show in Fig 2(e-g) the estimation of the ODF with the same approach but with an increasing number of samples. As expected the results exhibits successive estimation accuracy improvements and converge to the true ODF shape. Using this synthetic experiments, we observed that more signal samples than our high resolution sampling protocol negligibly influence the estimation precision (results not shown). 


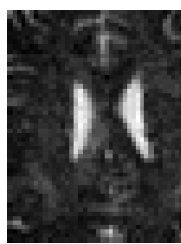

a) $S_{0}$

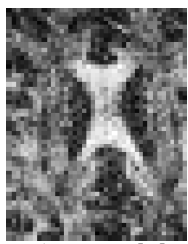

b) DTI 4

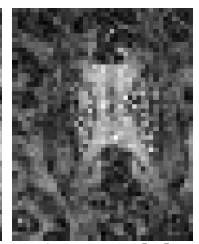

c) QBI 9]

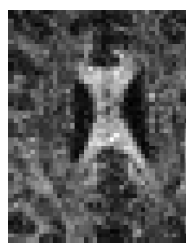

d) $\mathcal{G}=\mathrm{ODF}$

Fig. 3. Comparison of GA [7] on region of corpus callosum and lateral ventricles. Isotropic area are black, anisotropic area are white.

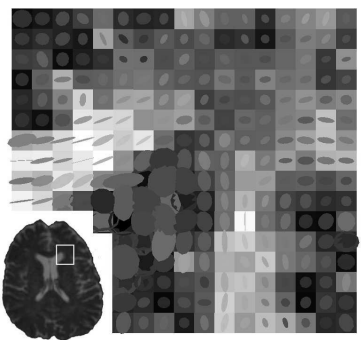

a) DTI 4

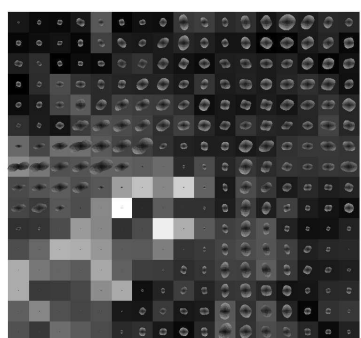

b) QBI 9

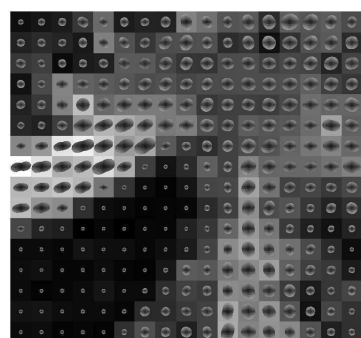

c) Our method, $\mathcal{G}=\mathrm{ODF}$

Fig. 4. In vivo brain white matter ODF overlaid on GA maps in a region of interest. $S_{0}$ image show the region of interest surrounding the corpus callosum-genu and the frontal horn, corpus callosum horizontal fibers appears from the left. DTI(a) and QBI(b) were computed with the outer shell $b=3000 \mathrm{~s} / \mathrm{mm}^{2}$. Our method (c) shows the ODF obtained from both shells $b=1000$ and $3000 \mathrm{~s} / \mathrm{mm}^{2}$.

In Vivo Experiments: Diffusion MR images were acquired in two shells along 32 directions at $b=1000 \mathrm{~s} / \mathrm{mm}^{2}$ and $b=3000 \mathrm{~s} / \mathrm{mm}^{2}$, and a single image at $b=0 \mathrm{~s} / \mathrm{mm}^{2}$. Thus, there were a total of 65 images acquired in a sequence of 15 minutes. The SENSE parallel imaging protocol was used with a factor of acceleration set to 2 ; and only $80 \%$ of the k-space was acquired. Matrix size was $112 \times 112 \times 60$ and the image resolution was $2 \times 2 \times 2 \mathrm{~mm}^{3}$. Repetition time was $\mathrm{TR}=11490 \mathrm{~ms}$, echo time was $\mathrm{TE}=85 \mathrm{~ms}$. Time between two pulses and time of diffusion gradients were respectively $\Delta=42.2 \mathrm{~ms}$ and $\delta=26.3 \mathrm{~ms}$. The probability maps were computed by following the procedure described in Fig 1 Terms up to $N=1$ and $L=4$ were used in the calculations. Computations were done in less than a minute on a $3 \mathrm{Ghz}$ processor, and includes calculations of SPF coefficients and projections on the single sphere along the 642 directions for the whole data-set $112 \times 112 \times 60$ volume.

We computed the generalized anisotropy (GA) measure [7, which is a generalization of the fractional anisotropy (FA) measure of DTI. We show results in Fig 3, each image was normalized independently to enhance visualization contrast. Representative images of the data are presented in Fig 4 and reveals microstructures around the genu of the corpus callosum. DTI performs well in corpus callosum but fails in voxel with orientational heterogeneity as shown in Fig 4. QBI can successfully retrieve multiple fibers orientations but is sensible 
to noise, especially in region of cerebrospinal fluid (c.f . Fig 4b). Besides, the QBI is a sharp approximation of the true ODF and thus enhances noise $(\operatorname{Fig}, 2(a, b))$. Whereas cerebrospinal fluid area are expected to exhibits isotropic diffusion, the ODF obtained by the QBI method exhibits anisotropy and may lead to misinterpretation of the brain structure on the GA map (Fig 3 ). On the contrary, the ODF obtained by our approach does not fall into this pitfall (Fig $3 \mathrm{~d}$ ), it successfully retrieves anisotropic shapes in brain white matter fibers regions and isotropic shape in cerebrospinal fluid area (c.f. Fig 4 4 ).

\section{Discussion and Conclusion}

In this paper, we provided a direct method for the estimation of various characteristics on the tissue microstructure in MR images. In a context where the origin of diffusion signal is still under study, this model-independent method uses in a fast and flexible manner the reduced number of signal samples. Additionally, we demonstrated the feasibility of our approach within clinical time-frame acquisition. Comparison with competing methods of the literature depicted our model in a favorable light.

\section{References}

1. Stejskal, E., Tanner, J.: Spin diffusion measurements: spin echoes in the presence of a time-dependent field gradient. Journal of Chemical Physics 42, 288-292 (1965)

2. LeBihan, D., Breton, E., Lallemand, D., et al.: Mr imaging of intravoxel incoherent motions: Application to diffusion and perfusion in neurologic disorders. Radiology, 401-407 (1986)

3. Callaghan, P.: Principles of Nuclear Magnetic Resonance Microscopy. Oxford University Press (1991)

4. Basser, P., Mattiello, J., LeBihan, D.: Estimation of the effective self-diffusion tensor from the nmr spin echo. J. Magn. Reson. 103, 247-254 (1994)

5. Jian, B., Vemuri, B.C., Özarslan, et al.: A novel tensor distribution model for the diffusion-weighted mr signal. NeuroImage 37, 164-176 (2007)

6. Wedeen, V., Reese, T., Tuch, D., et al.: Mapping fiber orientation spectra in cerebral white matter with fourier transform diffusion mri. In: ISMRM, p. 82 (2000)

7. Tuch, D., Weisskoff, R., Belliveau, J., Wedeen, V.: High angular resolution diffusion imaging of the human brain, p. 321 (1999)

8. Tuch, D.: Q-ball imaging. Magn. Reson. Med. 52, 1358-1372 (2004)

9. Descoteaux, M., Angelino, E., Fitzgibbons, S., Deriche, R.: Regularized, fast and robust analytical q-ball imaging. Magn. Reson. Med. 58, 497-510 (2007)

10. Yablonskiy, D.A., Bretthorst, G.L., Ackerman, J.J.: Statistical model for diffusion attenuated mr signal. Magn. Reson. Med. 50, 664-669 (2003)

11. Tournier, J., Calamante, F., Gadian, D., Connely, A.: Direct estimation of the fiber orientation density function from diffusion-weighted mri data using spherical deconvolution. NeuroImage 23, 1179-1185 (2004)

12. Liu, C., Bammer, R., Acar, B., Moseley, M.E.: Characterizing non-gaussian diffusion by using generalized diffusion tensors. Magn. Reson. Med. 51, 924-937 (2004) 
13. Özarslan, E., Sherperd, T.M., Vemuri, B.C., et al.: Resolution of complex tissue microarchitecture using the diffusion orientation transform (dot). NeuroImage 31, 1086-1103 (2006)

14. Assaf, Y., Basser, P.J.: Composite hindered and restricted model of diffusion (charmed) mr imaging of the human brain. NeuroImage 27, 48-58 (2005)

15. Wu, Y.C., Alexander, A.L.: Hybrid diffusion imaging. NeuroImage 36 (2007)

16. Ritchie, D.W.: High-order analytic translation matrix elements for real-space sixdimensional polar fourier correlations. J. Appl. Cryst. 38, 808-818 (2005)

17. Frank, L.: Characterization of anisotropy in high angular resolution diffusionweighted mri. Magn. Reson. Med. 47, 1083-1099 (2002)

18. Clark, C., Le Bihan, D.: Water diffusion and anisotropy at high b values in the human brain. Magn. Reson. Med. 44, 852-859 (2000)

19. Cohen, Y., Assaf, Y.: High b-value q-space analyzed diffusion-weighted mrs and mri in neuronal tissues - a technical review. NMR Biomed. 15, 516-542 (2002)

20. Niendorf, T., Dijkhuizen, R.M., Norris, D.G., van Lookeren, C.M.: Biexponential diffusion attenuation in various states of brain tissue: implications for diffusionweighted imaging. Magn. Reson. Med. 36, 847-857 (1996)

21. Assaf, Y., Cohen, Y.: In vivo and in vitro bi-exponential diffusion of n-acetyl aspartate (naa) in rat brain: a potential structural probe? NMR Biomed. 11 (1998)

22. Biedenharn, L.C., Louck, J.D.: Angular momentum in quantum physics. AddisonWesley Publishing Co., Reading (1981)

23. Blanco, M.A., Flórez, M., Bermejo, M.: Evaluation of the rotation matrices in the basis of real spherical harmonics. J. Mol. Struct. 419, 19-27 (1997) 